\title{
NUEVA METODOLOGÍA PARA OPTIMIZAR LA PROGRAMACIÓN DEL RIEGO APROVECHANDO LA DIFERENCIA DE COTA ENTRE EL PUNTO DE SUMINISTRO Y EL DE CONSUMO
}

\author{
Alonso Campos, J.C. (1) (P), Jiménez Bello, M.A. (2), \\ Martínez Alzamora, F. (3), Bou Soler, V. (4) \\ ${ }^{1}$ Ing. Industrial, becario investigación, Inst. U. Ingeniería del Agua y M.A (IIAMA), Universitat \\ Politècnica de València, Camino Vera, bq 5J, bajo 46022 Valencia, joaalcam@etsii.upv.es \\ ${ }^{2}$ Investigador Contratado, Inst. U. Ingeniería del Agua y Medio Ambiente (IIAMA), Universitat \\ Politècnica de València. Camino de Vera, bq 5J, bajo 46022.Valencia. mijibar@dihma.upv. \\ ${ }^{3}$ Cat. Ing. Hidráulica, Instituto de Ingeniería del Agua y M.A (IIAMA), Universitat Politècnica \\ de València, Camino Vera, bq 5J, bajo 46022 Valencia, fmartine@hma.upv.es \\ 4 Ing. Informático, Investigador Contratado, Inst. U. Ingeniería del Agua y M.A (IIAMA), \\ Universitat Politècnica de València, Camino Vera, bq 5J, bajo 46022 Valencia, \\ vibouso@dihma.upv.es
}

\section{Resumen}

El elevado consumo energético vinculado a la operación de redes de riego por goteo o aspersión, junto con el notable aumento de las tarifas eléctricas en los últimos años, ha propiciado el desarrollo de metodologías y estrategias orientadas a reducir el coste de explotación de dichas redes. Para las redes en las que el punto de suministro tiene una cota suficientemente elevada para alimentar algunas tomas en condiciones adecuadas de presión, puede obtenerse un importante ahorro energético si se aprovechan las horas en las que la electricidad es más cara para regar el máximo número de tomas posible sin hacer uso del bombeo. En el presente trabajo se ha desarrollado una metodología que maximiza el volumen aportado por gravedad en un determinado periodo de tiempo, a la vez que garantiza una presión mínima en cada punto de consumo, determinando para ello los instantes de inicio y fin de riego de cada parcela. La metodología se basa en la combinación de un Algoritmo Genético con la simulación hidráulica de la red en Epanet, y se ha implementado en el entorno de programación Visual Studio mediante el lenguaje de programación C\#. La optimización se ha aplicado a dos casos reales alcanzando un incremento del volumen aportado del orden del 30\% respecto de la situación actual.

\section{Abstract}

The high amount of energy required for the operation of pressurized irrigation networks, and the increasing electric tariffs over the last years have led researchers to develop new methodologies and strategies focused on reducing the exploitation cost of these networks. Among irrigation networks there are many in which the head of the source node is high enough to deliver water to some takeoffs at the required pressure. For these cases, considerable savings can be obtained by performing the irrigation of the maximum number of takeoffs without pumping during the hours when energy is more expensive. In this work a new methodology was developed for maximizing the volume delivered by gravity during a specific time interval, while guaranteeing a minimum service pressure at each operating takeoff, by setting the instants when each takeoff starts and finishes irrigation. The methodology is based on combining a Genetic Algorithm with the hydraulic simulation of the network using the Epanet toolkit. It was developed in Visual Studio by means of the C\# 
programming language. The optimization was tested on two real cases and it was achieved a volume increase delivered by gravity of around $30 \%$ compared to the current situation.

\section{1- Introducción}

El proceso de modernización de los sistemas de riego en España ha acarreado un aumento del consumo energético en el sector del 1.900\% entre 1950 y 2007 (Corominas 2010). Los sistemas de riego localizado y por aspersión permiten un ahorro en el consumo de agua pero precisan presiones de trabajo elevadas que suponen un consumo de energía mayor que los sistemas de riego por superficie (Santos et al. 2010, Rodríguez-Díaz et al. 2011).

Cuando el modo de operación de una red de riego es a la demanda, en general se han de sobredimensionar los elementos para prever las mayores pérdidas por fricción debido a la existencia de picos de demanda. Se ha demostrado que se pueden alcanzar ahorros considerables sustituyendo el sistema de riego a la demanda por un riego organizado por turnos (Rodríguez Díaz et al. 2009, Moreno et al. 2010, Jiménez-Bello et al. 2010, Jiménez-Bello et al. 2011, Carrillo Cobo et al. 2011, Navarro Navajas et al. 2012, Fernández García et al. 2013).

El resultado de sectorizar una red de riego desde el punto de vista del ahorro energético pasa por establecer tiempos de riego iguales para todas las parcelas de cada sector. Este hecho puede ser precursor de ineficiencias, puesto que el tiempo de riego necesario para cada una es generalmente distinto, y se calcula en base a las necesidades de riego de cada cultivo y al caudal base de la toma. Por ello, una alternativa a sectorizar la red es obtener una programación completa de la los instantes de apertura y cierre de cada toma de manera que se minimice el consumo energético y al mismo tiempo se ajusten los tiempos de riego (Jiménez-Bello et al. 2013).

Por otra parte, las tarifas eléctricas de 3 o 6 periodos generalmente contratadas por las comunidades de regantes, presentan importantes diferencias de precio entre las horas valle y punta, pudiendo obtener considerables ahorros económicos si se evita activar el bombeo en las horas en que la energía es más cara. Un primer paso a tener en cuenta a la hora de generar una programación de riego es comprobar si en los puntos de suministro existe suficiente energía potencial (cota) para poder efectuar el riego de alguna parcela en condiciones adecuadas de presión sin el auxilio del bombeo. En estos casos pueden aprovecharse las horas en que la energía es más cara para regar dichas parcelas.

En el presente trabajo se ha desarrollado una metodología para la obtención de la programación de riego que maximiza el volumen de agua entregado sin aporte de energía durante un intervalo de tiempo predefinido.

\section{2- Metodología}

Dada una red de riego, con un número de puntos de suministro $\mathrm{N}_{\mathrm{s}}$, y un número de tomas $\mathrm{N}_{\mathrm{t}}$, se considerarán variables del problema aquellas tomas cuya diferencia de cota respecto del punto de suministro más elevado sea mayor que la presión mínima requerida de funcionamiento $P_{\min }$ (m.c.a). Se tienen por tanto un número de variables $0<N_{V}<=N_{t}$. Cada toma tendrá definido un caudal base $Q_{i}$ que se considerará constante siempre que la toma esté abierta, y un tiempo de riego $\mathrm{T}_{\text {ri }}$.

Se establece un intervalo de tiempo destinado al riego de las tomas sin aporte energético $\left(T_{\text {grav }}<24 \mathrm{~h}\right.$ ), y se discretiza en $\mathrm{N}_{\text {int }}$ intervalos de igual duración. El problema de optimización para un único intervalo de cálculo se plantea del siguiente modo:

$$
\max f\left(x_{1}, x_{2}, \ldots, x_{N_{v}}\right)=\sum_{i=1}^{N_{V}} x_{i} \cdot Q_{i}
$$

Sujeto a: 


$$
\mathrm{Pi}>\text { Pmin si Qi > } 0
$$

La ecuación 1 es la función objetivo. Las variables $x_{i}$ toman valores binarios $(0,1)$ que indican si la toma está abierta o cerrada. Cada toma abierta deberá disponer de presión suficiente para su correcto funcionamiento.

Una vez obtenido el resultado para un instante dado, las tomas que han resultado activadas permanecen en ese estado hasta que finaliza su tiempo de riego. El siguiente intervalo de tiempo en el que se efectuará la optimización coincide con el instante en que finaliza su tiempo de riego al menos una de las tomas activas. El proceso se repite hasta llegar al último intervalo de tiempo o bien todas las tomas consideradas han finalizado su tiempo de riego. Hay que destacar que en cada iteración del algoritmo el número de variables disminuye, puesto que dejan de considerarse las tomas que han iniciado el riego.

El proceso de optimización se ha programado en la plataforma .NET, apoyado en la simulación del modelo matemático de la red mediante Epanet (Rossman 2000), y en la implementación de un Algoritmo Genético (Goldberg \& Kuo 1987), desarrollado sobre la base de la librería Aforge.Genetic.

La metodología expuesta se ha aplicado en dos casos de estudio. Puesto que se dispone de la programación actual en ambos casos, como paso previo se ha partido de la simulación de dichas programaciones y se han comparado los resultados con los obtenidos mediante la presente metodología. Por último se han comparado estos resultados con los obtenidos mediante la metodología descrita en Jiménez-Bello et al. 2013. Este método, basado también en la combinación de un Algoritmo Genético con la simulación hidráulica en Epanet, define las variables como el instante en el que cada toma inicia el riego. Por lo tanto, el número de variables coincide con el número inicial de variables del nuevo método, siendo en este caso las variables enteras.

\section{3- Casos de estudio}

\section{1- Realón}

El primer caso de estudio es la red de riego de "Realón" en Picassent (Valencia) (Figura 1). La red cuenta con 62 hidrantes multiusuario con un total de 362 tomas y una balsa de regulación en cabecera. Se ha establecido una presión mínima de 25 mca en todas las tomas. El número de tomas cuya diferencia de cota con la balsa es mayor de $25 \mathrm{~m}$ es de 191 (número inicial de variables). El periodo de tiempo destinado al riego sin aporte energético es de 14 horas (de 10:00 a 24:00), y se ha discretizado en intervalos de 5 minutos. 


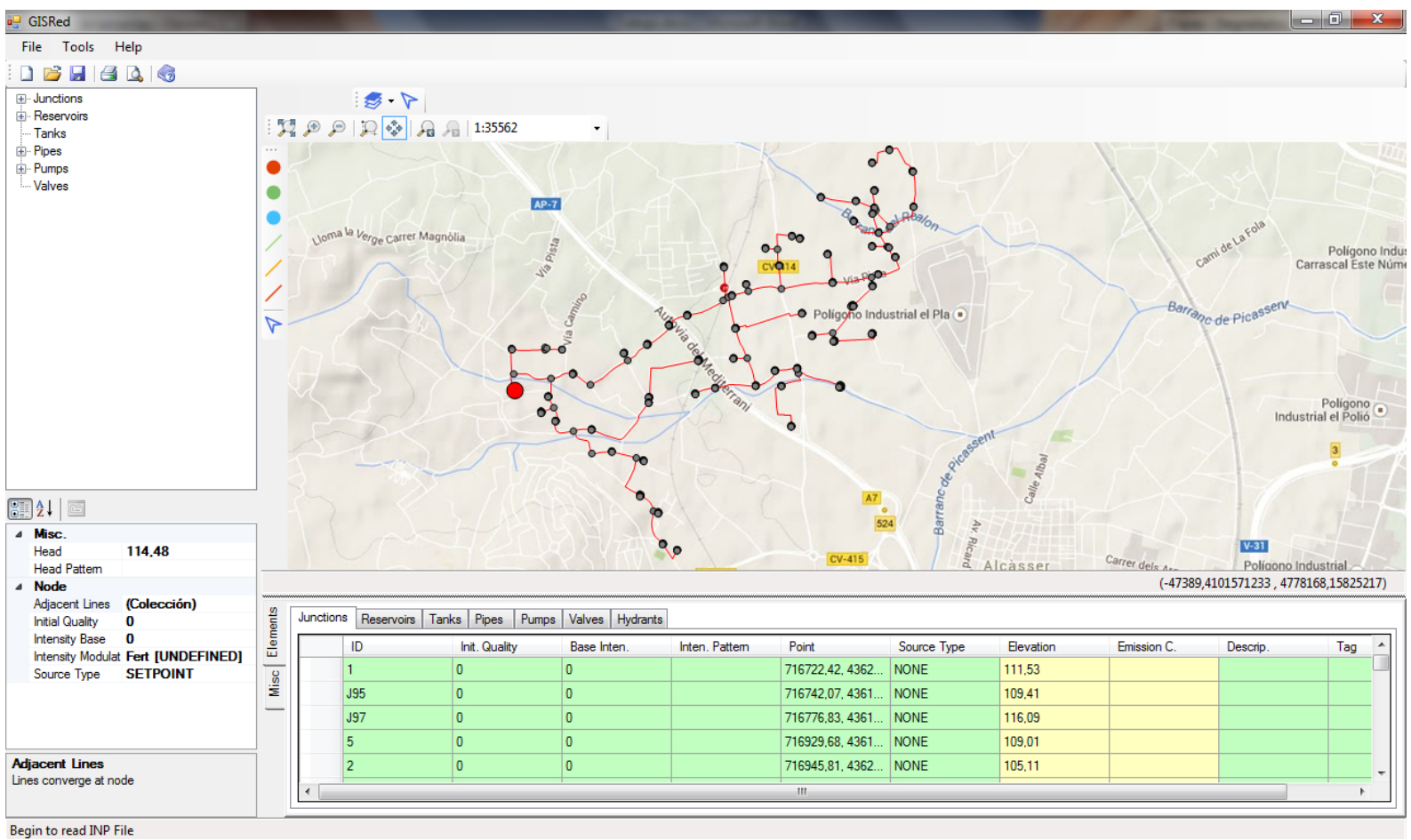

Figura 1. Red de riego de Realón

\section{2- Camí de Torrent}

La red de riego "Camí de Torrent" en Picassent (Valencia) constituye el segundo caso de estudio (Figura 2). Se trata de una red de características similares a la de Realón, en este caso con 68 hidrantes y un total de 327 tomas, de las cuales 247 han sido consideradas como variables del problema. Se han definido los parámetros temporales idénticos a los de la red de Realón. En ambos casos son redes actualmente operadas según una programación definida por el personal responsable de la operación, mediante criterios basados en la homogeneización del caudal total. Las presiones mínimas de servicio se garantizan mediante prueba y error. 


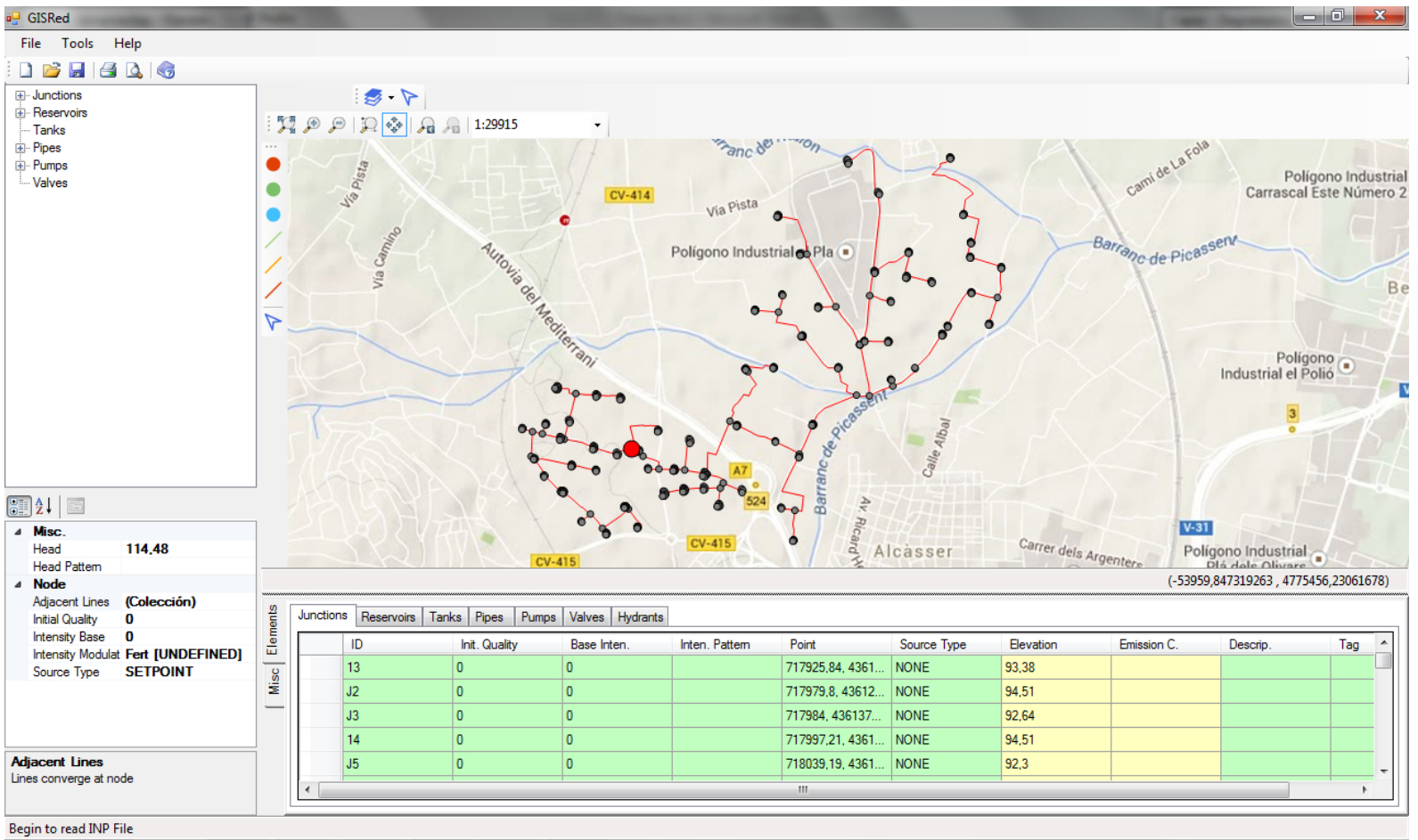

Figura 2. Red de riego Camí de Torrent

\section{4- Resultados}

Los resultados presentados en la Figura 3 se corresponden con el análisis de la programación actual. Se concluye que la presión mínima de servicio en cada punto de consumo (25 m.c.a.) en general no está garantizada en todo momento. En total hay 20 tomas en el caso de Realón y 50 en el caso de Camí de Torrent que presentan una presión inferior a la mínima de servicio. Por otra parte el volumen total entregado a lo largo de las 14 horas de riego sin aporte de energía es de $2.172 \mathrm{~m}^{3}$ en la red de Camí de Torrent y de 1.635 $\mathrm{m}^{3}$ en la de Realón. 

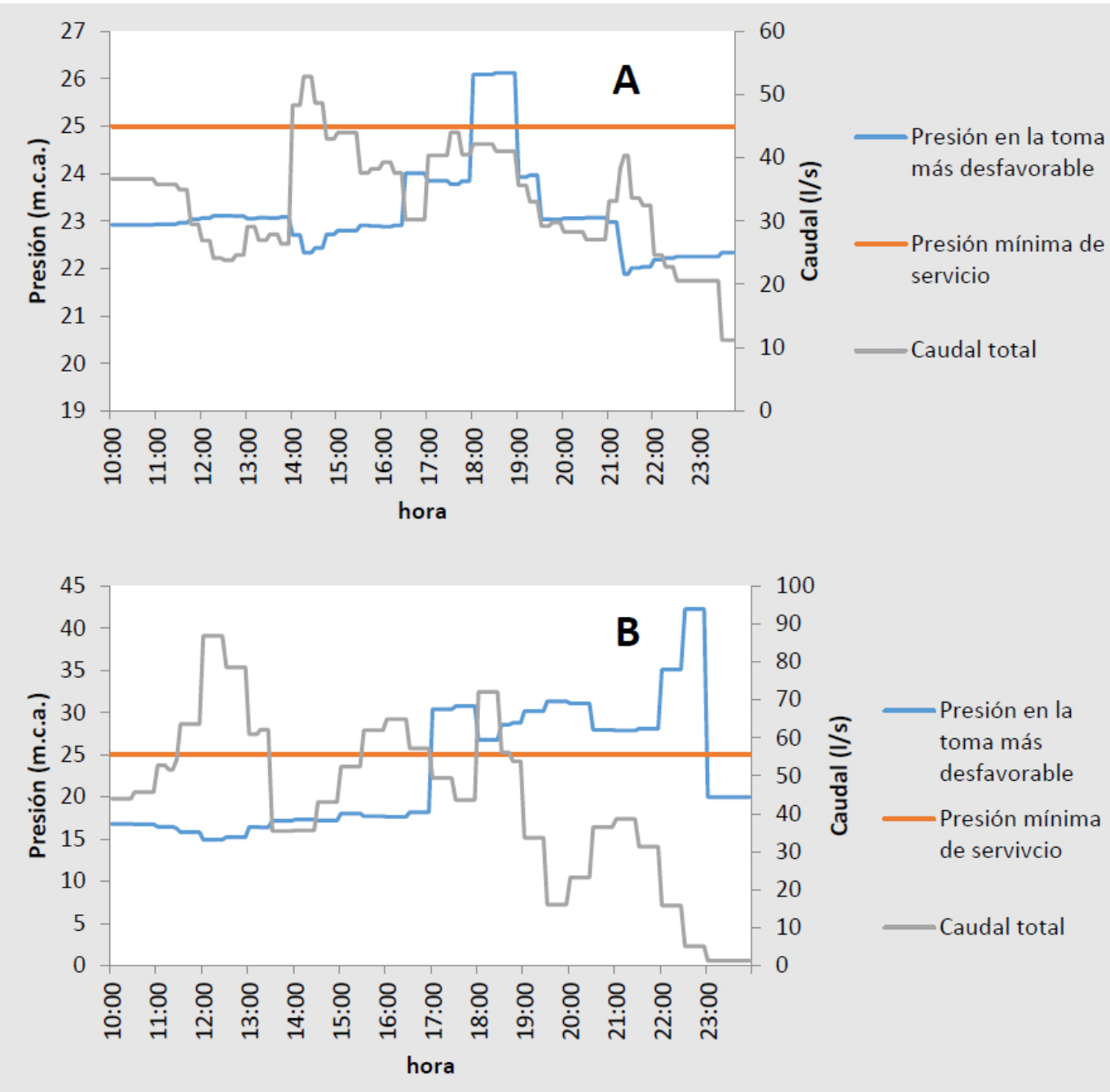

Figura 3. Resultados de simular la programación de riego actual. Red de Realón (A). Red de Camí de Torrent (B)

En cuanto a los resultados obtenidos siguiendo la metodología propuesta en Jiménez-Bello et al. 2013 (Figura 4) se ha alcanzado un suministro de $2.514 \mathrm{~m}^{3}$ en el caso de Realón y de $2.864 \mathrm{~m}^{3}$ en el caso de Camí de Torrent. En ambos casos todas las tomas activas presentan una presión igual o superior a la mínima de servicio. El tiempo de cálculo de las soluciones presentadas es del orden de 2 - 3 horas ${ }^{1}$.

\footnotetext{
${ }^{1}$ Cálculos realizados en un ordenador ACER Aspire 5920G, procesador Intel Core 2 Duo a 2 $\mathrm{GHz}, 4 \mathrm{~GB}$ de RAM.
} 


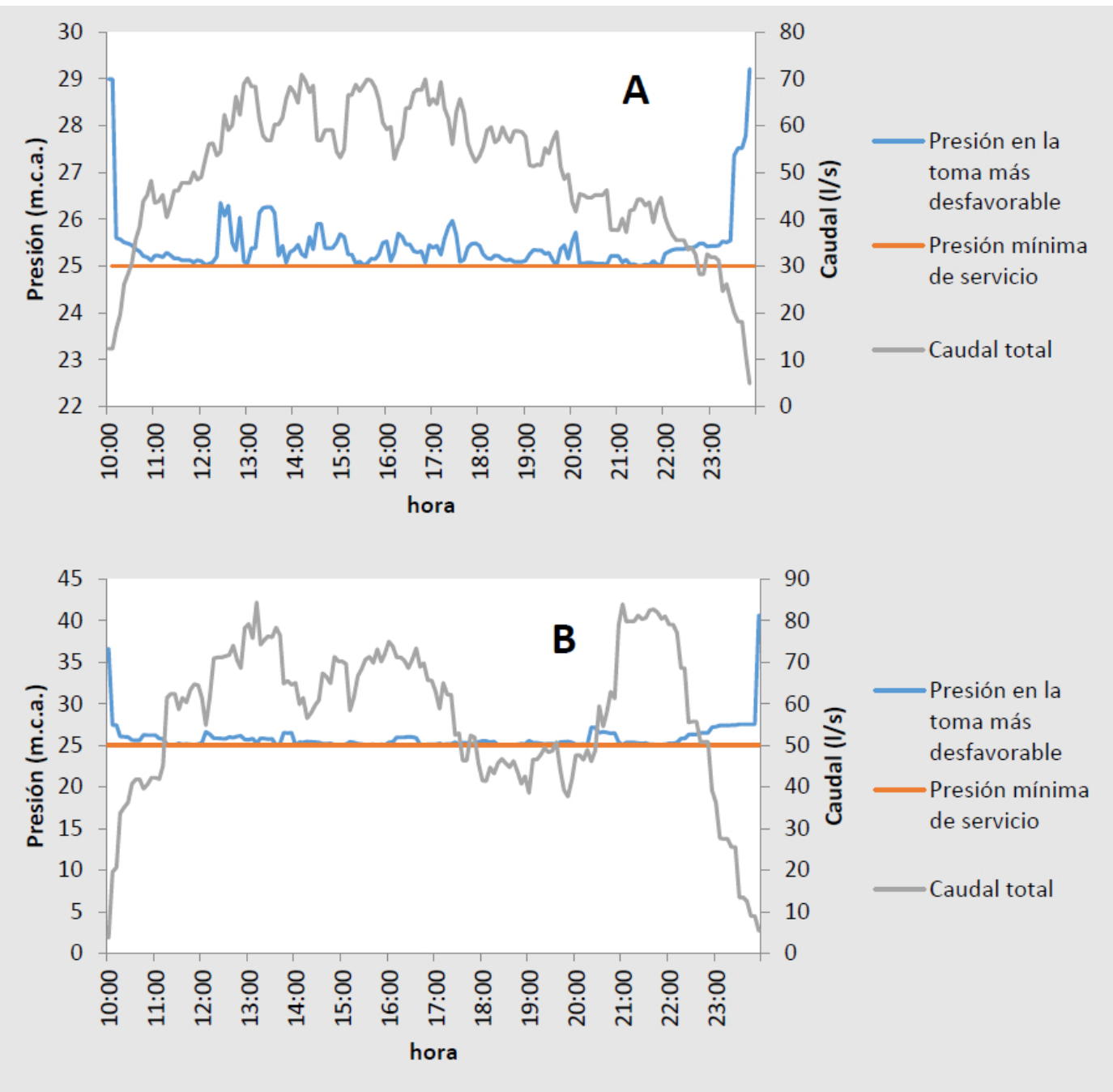

Figura 4. Resultados optimización mediante la metodología descrita en (Jiménez-Bello et al. 2013). Red de Realón (A). Red de Camí de Torrent (B)

Siguiendo el método expuesto en el presente trabajo se ha logrado suministrar un volumen de 2.517,2 $\mathrm{m}^{3}$ en la red de Realón y de 2.908,4 $\mathrm{m}^{3}$ en Camí de Torrent a lo largo de las 14 horas de riego sin bombeo. Este valor supone un aumento del 53,95\% y del $30,37 \%$ respecto de la situación actual, y un ligero aumento respecto del resultado obtenido mediante el método anterior. Se observa en los resultados que en la metodología anterior el caudal total es más homogéneo a lo largo de la jornada, mientras que la nueva metodología tiende a concentrar mayores caudales al principio del periodo de optimización.

El volumen máximo teórico que podría suministrarse por gravedad es de $2.673 \mathrm{~m}^{3}$ para el caso de Realón, y de $3.004 \mathrm{~m}^{3}$ para el de Camí de Torrent. Por lo tanto se ha alcanzado un $94,1 \%$ y un $96,8 \%$ respectivamente del máximo potencial.

El aumento del volumen suministrado por gravedad conllevará una reducción del volumen bombeado en las horas nocturnas, con el consiguiente ahorro energético. A su vez la reducción del caudal bombeado permitirá disminuir la presión de consigna debido a las menores pérdidas de carga en la red. Finalmente se puede obtener aún otro ahorro adicional si se consigue que la estación de bombeo trabaje en todo momento en puntos de funcionamiento eficientes.

Además, mediante esta metodología se ha garantizado en todo momento la presión mínima en las tomas activas (Figura 5). 
Por otra parte, si se analiza la curva de caudal total se puede concluir que las tomas que se activan en último lugar son las más restrictivas en cuanto a la penalización de la presión de funcionamiento. Por lo tanto, la presente metodología aporta información acerca de los puntos críticos de la red.

El tiempo de cálculo empleado para optimizar el riego por gravedad ha sido inferior a 15 minutos. Esto supone dividir por 10 el tiempo necesario mediante la anterior metodología, con lo que el nuevo método podría ser de aplicabilidad en la operación de redes en tiempo real.

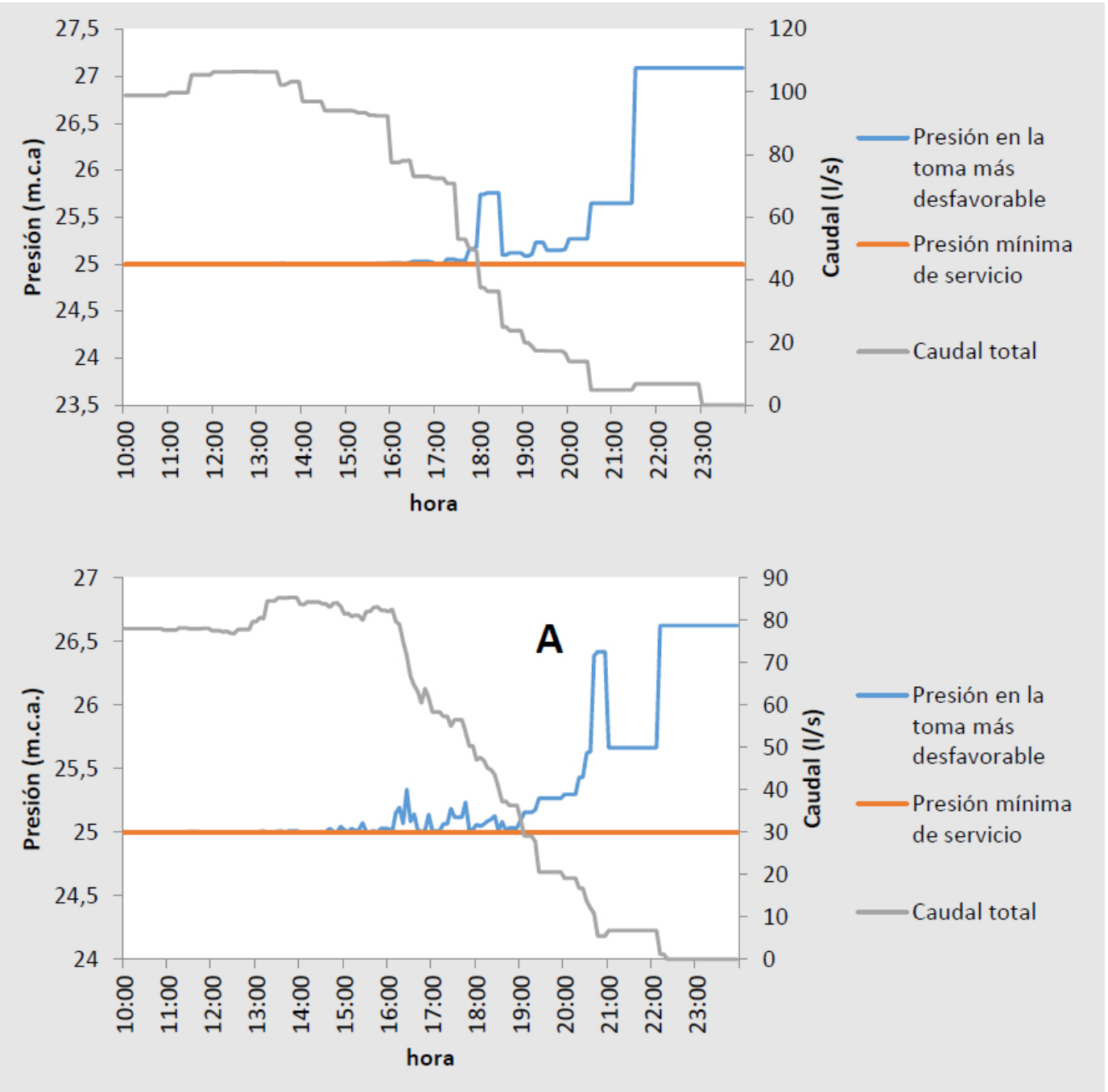

Figura 5. Resultados optimización mediante la nueva metodología. Red de Realón (A). Red de Camí de Torrent (B)

\section{5- Conclusiones}

Se ha desarrollado una metodología para la obtención de una programación de riego que maximiza el volumen total entregado sin aporte de energía en un determinado intervalo de tiempo, a la vez que garantiza una presión mínima en las tomas activas.

Siguiendo el método expuesto se han obtenido resultados para dos casos de estudio que incrementan el volumen entregado alrededor de un $30 \%$, presentando presiones de trabajo mayores que la deseada en cada instante. 
Con esta nueva metodología se ha logrado reducir el tiempo de cálculo a una magnitud que lo hace adecuado para la optimización en tiempo real de redes de riego.

Los resultados aportados por el algoritmo ofrecen implícitamente información sobre los puntos más críticos de la red.

\section{6- Agradecimientos}

Este trabajo ha sido financiado por el Proyecto Europeo FIGARO (Flexible and preclse irriGation plAtform to improve faRm scale water prOductivity), dentro del $7^{\circ}$ Programa Marco, en el ámbito "Precision technologies to improve irrigation management and increase water productivity in major water-demanding crops in Europe" (KBBE.2012.1.1-03).

\section{7- Bibliografía}

Carrillo Cobo, M.T. et al., 2011. Low energy consumption seasonal calendar for sectoring operation in pressurized irrigation networks. Irrigation Science, 29(2), pp.157-169.

Corominas, J., 2010. Agua y Energía en el riego en la época de la sostenibilidad. Ingeniería del Agua, 17(3).

Fernández García, I. et al., 2013. Optimal Operation of Pressurized Irrigation Networks with Several Supply Sources. Water Resources Management, 27(8), pp.2855-2869.

Goldberg, D.E. \& Kuo, C.H., 1987. Genetic Algorithms in Pipeline Optimization. Journal of Computing in Civil Engineering, 1(2), pp.128-141.

Jiménez-Bello, M. a. et al., 2010. Methodology for grouping intakes of pressurised irrigation networks into sectors to minimise energy consumption. Biosystems Engineering, 105(4), pp.429-438.

Jiménez-Bello, M.A. et al., 2013. A methodology to improve water and energy use by network sectoring and proper irrigation scheduling. In 7th IWA Intenational Conference on Efficient Use and Management of Water. Paris, pp. 1-11.

Jiménez-Bello, M.Á. et al., 2011. Validation of a methodology for grouping intakes of pressurized irrigation networks into sectors to minimize energy consumption. Agricultural Water Management, 102(1), pp.46-53.

Moreno, M.A. et al., 2010. Energy efficiency of pressurised irrigation networks managed ondemand and under a rotation schedule. Biosystems Engineering, 107(4), pp.349-363.

Navarro Navajas, J.M. et al., 2012. Impacts of irrigation network sectoring as an energy saving measure on olive grove production. Journal of Environmental Management, 111, pp.1-9. Available at: http://dx.doi.org/10.1016/j.jenvman.2012.06.034.

Rodríguez Díaz, J.A. et al., 2009. Exploring energy saving scenarios for on-demand pressurised irrigation networks. Biosystems Engineering, 104(4), pp.552-561.

Rodríguez-Díaz, J.A. et al., 2011. The paradox of irrigation scheme modernization: more efficient water use linked to higher energy demand. Spanish Journal of Agricultural Research, 9(4), pp.1000-1008. 
XXXIII Congreso Nacional de Riegos

Universitat Politècnica de València, Valencia 2015

DOI:http://dx.doi.org/10.4995/CNRiegos.2015.1518

Rossman, L. a, 2000. EPANET 2: users manual. Cincinnati US Environmental Protection Agency National Risk Management Research Laboratory, 38(September), p.200.

Available at: http://nepis.epa.gov/Adobe/PDF/P1007WWU.pdf.

Santos, L. et al., 2010. El Riego Y Sus Tecnologías Europa-Amé., Lisboa. 